Adrian F. Fernando, MD Joselito F. David, MD 1,2,3,4

'The Head \& Neck Reconstructive Surgery Fellowship Consortium,

Department of Otorhinolaryngology Head \& Neck Surgery

Jose R. Reyes Memorial Medical Center Manila, Philippines

\section{${ }^{2}$ Department of Otorhinolaryngology} Head \& Neck Surgery

East Avenue Medical Center

Quezon City, Philippines

${ }^{3}$ Department of Otorhinolaryngology Head \& Neck Surgery

Jose R. Reyes Memorial Medical Center

Manila, Philippines

${ }^{4}$ Department of Otorhinolaryngology Head \& Neck Surgery

University of Sto. Tomas Hospital

Manila, Philippines

\title{
Combination of Autologous Platelet-Rich Fibrin and Bone Graft: An Invaluable Option for Reconstruction of Segmental Mandibular Defects
}

\section{Dear Editor,}

Reconstruction of mandibular defects resulting from ablative surgery for benign and malignant tumors remains a reconstructive challenge. For the past decade, the fibular free flap has been the workhorse for large mandibular defects because of its length, versatility and ability to be harvested with a skin paddle for soft tissue closure. Although its success rate has continuously improved to almost $95 \%$, donor site morbidity remains a matter of concern. ${ }^{1,2}$ Bone grafts are already widely used in dental surgery but only as fillers for chipped or marginal defects and not for large segmental mandibular defects. We present a new technique of reconstructing segmental mandibular defects using bone grafts combined with autologous platelet-rich fibrin (PRF), a biomaterial derived intra-operatively from the patient that incorporates leukocytes, platelets, growth factors, and a wide range of glycoproteins in a dense fibrin matrix. Moreover, we describe the essential role of PRF in bone healing and regeneration that offers an invaluable reconstructive option that is free of donor site morbidity without sacrificing the main goal of reconstruction in restoring both form and function.

Keywords: Mandibular reconstruction, segmental mandibular defect, bone graft, autologous Platelet-Rich Fibrin (PRF)

\section{MATERIALS AND METHODS}

Correspondence: Dr. Adrian F. Fernando The Head \& Neck Reconstructive Surgery Fellowship Consortium

Department of Otorhinolaryngology - Head \& Neck Surgery

4/F Jose R. Reyes Memorial Medical Center

San Lazaro Compound, Rizal Avenue, Manila 1003

Philippines

Phone: (632) 7119491 local 320

Fax: (632) 7436921

E-mail: ianfernando_md@yahoo.com

Reprints will not be available from the author.

The authors declared that this represents original materia that is not being considered for publication or has not been published or accepted for publication elsewhere, in full or in part, in print or electronic media; that the manuscript has been read and approved by all the authors, that the requirements for authorship have been met by each author, and that each author believes that the manuscript represents honest work.

Disclosures: The authors signed disclosures that there are no financial or other (including personal) relationships, intellectual passion, political or religious beliefs, and institutional affiliations that might lead to a conflict of interest.

\section{Subject and Indications}

A 23-year-old male underwent reconstruction with allogenic bone graft in combination with autologous platelet-rich fibrin (PRF) for a large segmental angle to parasymphyseal mandibular defect. (Figure 1) In this example, the authors' technique for segmental mandibular defect reconstruction using bone grafts with PRF was best performed as a second stage procedure following tumor ablation to prevent contamination from oral cavity secretions. As with any elective procedure, a thorough review of the medical history, control of systemic disease and informed consent were necessary. Patients with contraindications for fibular free flaps, such as history of peripheral vascular disease, unfavorable imaging of the lower extremity, venous insufficiency, and anomalous lower extremity vasculature may benefit from this technique. ${ }^{3}$ Patients who had failed mandibular reconstruction with other methods such as those reconstructed with single alloplastic material, titanium plate, non-vascularized autologous bone graft or free flaps are likewise candidates for this option. 


\section{LETTER TO THE EDITOR}

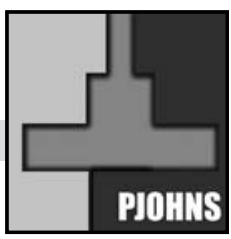

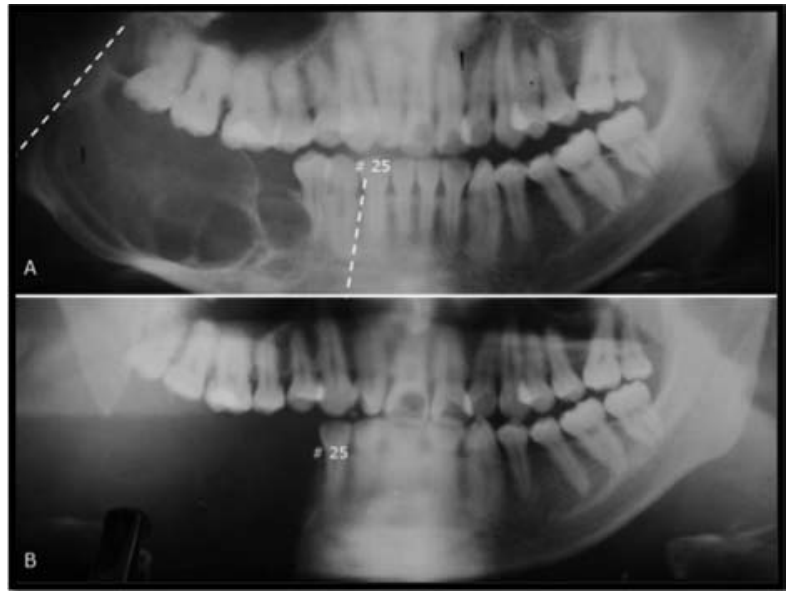

Figure 1. Ameloblastoma. (A) Panoramic radiograph of a 23-year old male who underwent segmental mandibuletomy (broken lines) for ameloblastoma. (B) 10 days post-resection imaging of the mandible.

\section{Procedure}

A submandibular incision was made and a sub-platysmal flap was raised to expose the entire length of the mandibular defect. Apart from the preservation of vital structures in the area, it was important not to violate the oral mucosa to prevent contamination of the reconstruction site. A cortico-cancellous allogenic bone graft (Maxgraft $^{\circledR}$, Botiss Medical AG, Berlin, Germany) was fitted to the mandibular defect and anchored with bicortical screws $(2.5 ; 2.8 \mathrm{~mm}$, lengths $14-20 \mathrm{~mm})$ to a pre-bended $2.5 \mathrm{~mm}$ reconstruction titanium plate (Modus Reco 2.5, Medartis, Hochbergerstrasse Basel Switzerland). The remaining gaps between the inlaid grafts were filled with the remaining cancellous bone and biomimetic composite materials. (Figure 2) Although allogenic bone grafts with cortical and cancellous components are recommended for mandibular

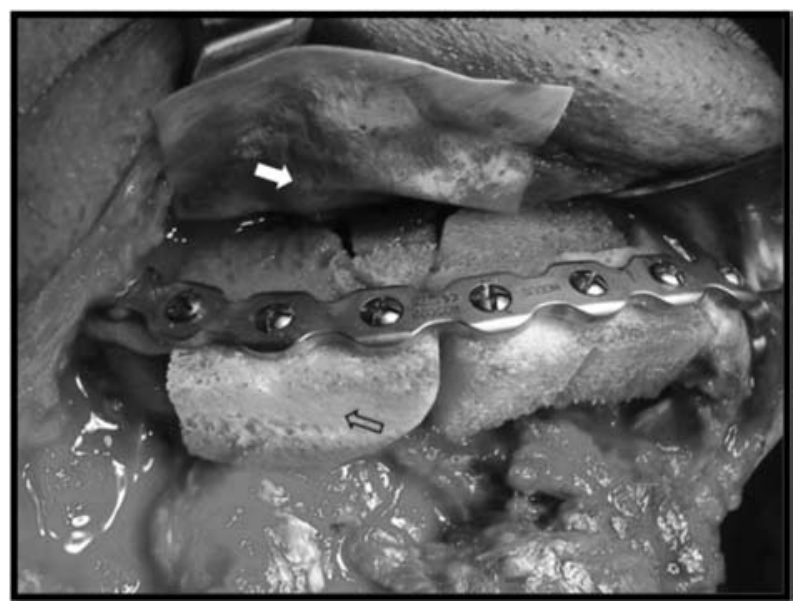

Figure 2. Anchoring of Bone Grafts. Mandibular reconstruction with cortico-cancellous bone grafts (open arrow) anchored to reconstruction plate and covered with a collagen membrane material (bold arrow). ramus and condylar reconstruction as these regions are composed of nearly $100 \%$ cortical bone, xenogenic (Cerabone ${ }^{\circledR}$, Botiss Medical AG , Berlin, Germany) or combined alloplastic material- (Maxresorb ${ }^{\circledR}$, Botiss Medical AG , Berlin, Germany) bone grafts may be used in other regions. (Figure 3)

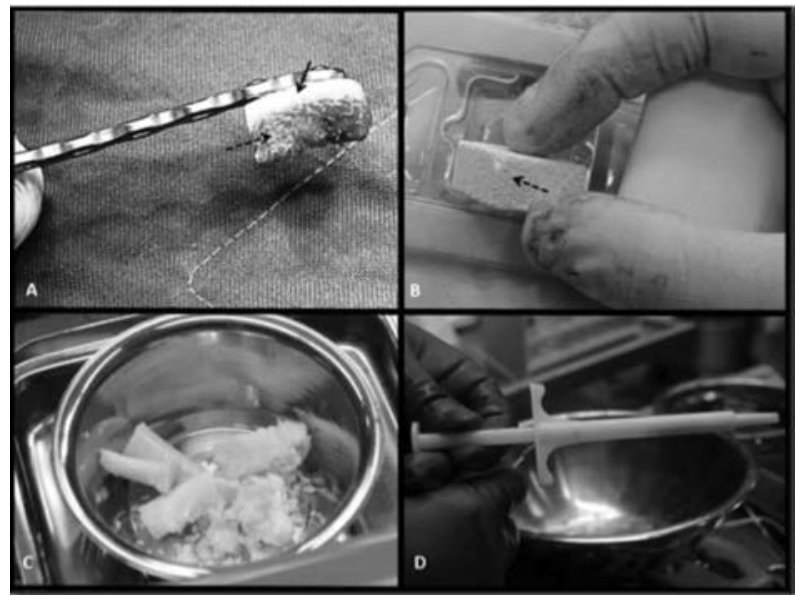

Figure 3. Different bone graft materials. (A) Human or allogenic cortico-cancellous bone graft (broken arrow); (B) Xenogenic bone graft; (C\&D) Biomimetic composites xenogenic bones that may be crushed or injected.

Meanwhile, venous blood was simultaneously drawn from the patient and placed in a $10 \mathrm{cc}$ glass collecting tube for single centrifuge processing using a PC-O2 centrifuge (PC-O2, Process, Nice, France). The specific centrifuge processes eight uncoated tubes using a standard protocol specially manufactured for processing PRF using $33^{\circ}$ tube angulation at 2700 RPM's, soft spin for 12 minutes. ${ }^{4}$ (Figure 4) At the end of the centrifugation process, three distinct fractions of blood components were produced where the intermediate fraction composed of dense PRF clot was used. The other blood components separated by the centrifugation process- serum or platelet-poor plasma (PPP)

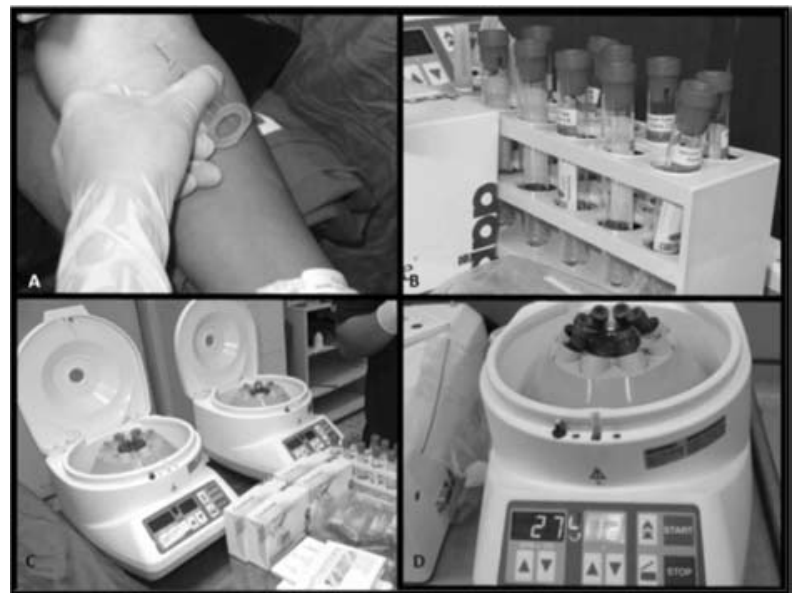

Figure 4. Blood centrifugation. (A) Intra-operatively extracted venous blood are placed in (B) plain $10 \mathrm{cc}$ collecting tubes for centrifuge with the (C\&D) The PC-O2 unit (PC-O2, Process, Nice, France) for PRF processing. 
and red blood cell concentrates, were respectively situated in the superficial and bottom layers of the collecting tube. The PRF clots were then transferred to a PRF processing box (PRF Box ${ }^{\oplus}$, Process, Nice, France) to prepare standardized membranes and harvest the PRF exudates in a sterile environment. ${ }^{5}$ (Figure 5) Collagen material of native pericardium (GTR/GBR) membrane (Jason ${ }^{\circledR}$ membrane, Botiss Medical AG, Berlin, Germany) was placed underneath the graft recipient site and the processed PRF membranes were layered over the graft recipient site to stimulate osteoblastic differentiation and neoangiogenesis. ${ }^{6}$

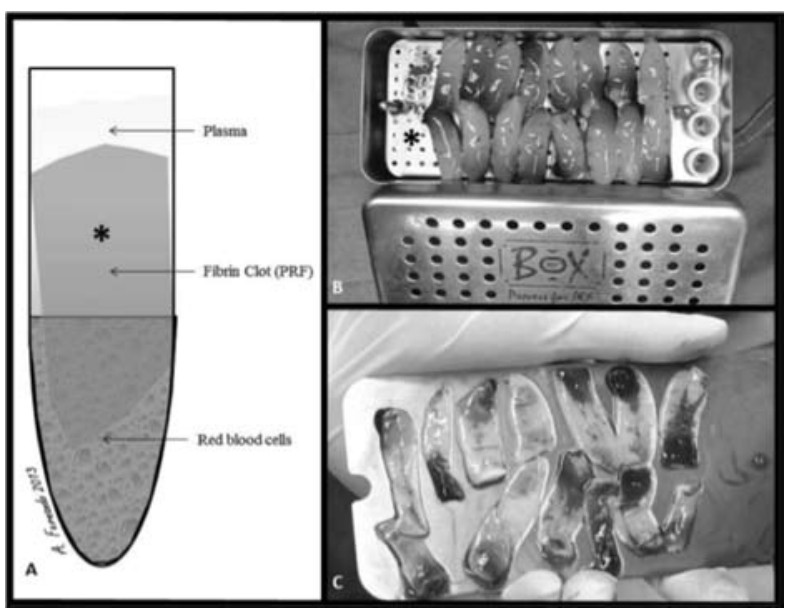

Figure 5. Processing of PRF. (A) Diagram of a harvested blood after centrifugation using the $\mathrm{PC}-\mathrm{O} 2$ protocol wherein the intermediate fibrin clot layer $\left(^{*}\right)$ is processed in a sterile environment $(B)$ to separate the fibrin clot and other blood components to produce $(C)$ leukocyte-rich PRF membranes.

The entire recipient site was then enveloped with the collagen material, mechanically securing the autologous PRF in contact with the bone grafts. (Figure 6) This established a membrane barrier for guided bone regeneration (GBR) and guided tissue regeneration (GTR) by preventing growth of undesired cells inside

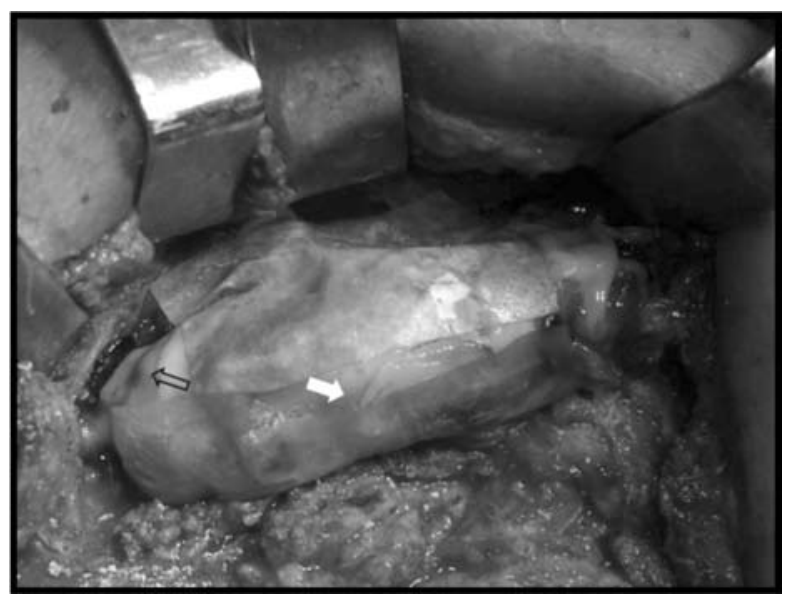

Figure 6. Establishing a membrane barrier. The entire recipient site is enveloped with native pericardium membranes (bold arrow) to mechanically secure the autologous PRF (blank arrow) in contact with the bone grafts. the neomandible and allowing osteogenesis and angiogenesis.? Commercially available collagen biomaterials vary from native collagen membrane to enhanced $\mathrm{Ca} / \mathrm{P}$ collagen composite materials such as the 3D-stable collagen graft (Mucoderm ${ }^{\oplus}$, Botiss Medical AG, Berlin, Germany) with larger available sizes for long mandibular defect coverage. The skin was closed in the usual manner and the patient was initially maintained on a liquid diet, progressing to a soft diet over 2-4 weeks. Plain panoramic radiographs after a week confirmed proper alignment of the bone grafts and monthly radiographic series was recommended for the first 6 months after reconstruction.

\section{RESULTS}

Monthly panoramic radiographs for the first six months after reconstruction showed absence of bone resorption. A $3 D$ reconstruction $C T$ imaging of the mandible was done after 10 months for placement of three osteo-integrated dental implants. (Figure 7) Bone biopsies were also taken in conjunction with placement of dental implants, and sent to the University of Bonn, Germany for histologic evaluation. (Figure 8) The trichromestained specimens showed new mineralized tissues consisting of woven bone characterized by high numbers of distributed osteocytes and irregularly arranged fiber bundles within the new bone matrix confirming bone regeneration. (Figure 9) The latest panoramic radiograph of the patient at 26 months after surgery showed absence of gaps between the bone grafts and their junction with the normal mandible, evincing complete bone regeneration and a successful mandibular reconstruction. (Figure 10) A total of 14 cases of segmental mandibular defects have been reconstructed by the authors using the particular technique from January 2011 to February 2013 with 100\% success rate and will be reported as a series in the near future.

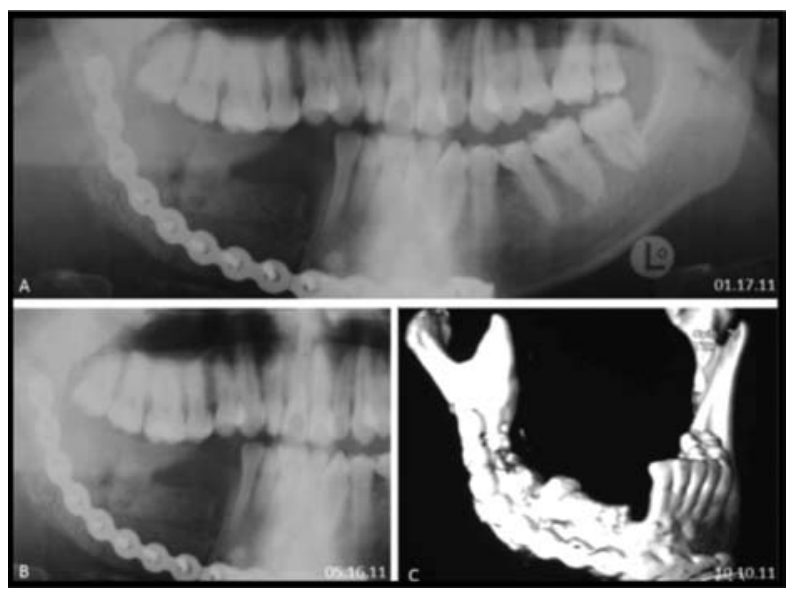

Figure 7. Radiographic series. (A) Plain panoramic radiograph 10 days after the procedure showing proper alignment of biomaterials. Plain radiograph (B) and 3D CT imaging (C) of the neomandible 4 and 9 months after respectively shows absence of bone resorption. 


\section{LETTER TO THE EDITOR}

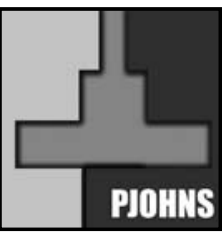

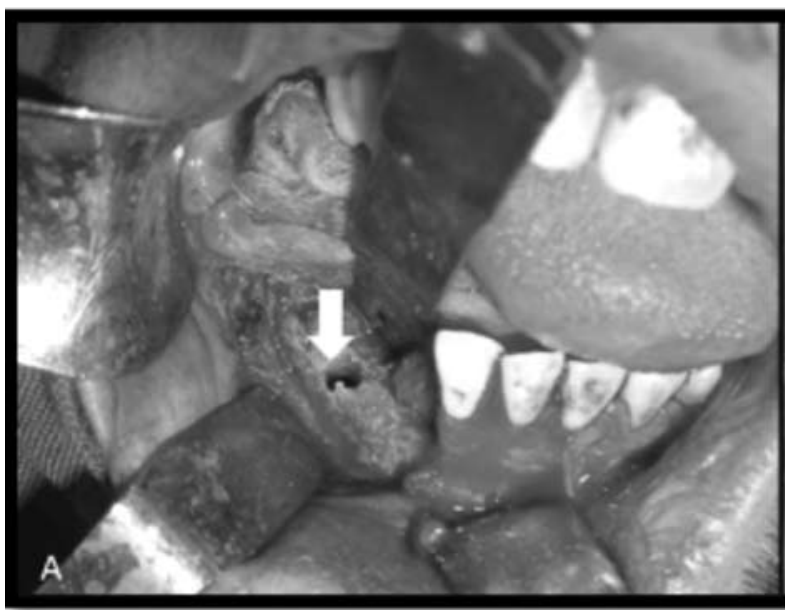

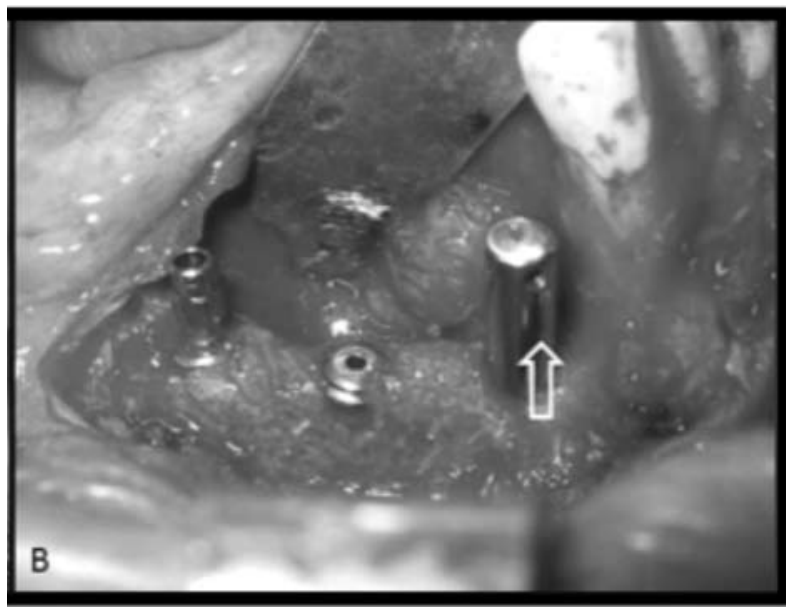

Figure 8. Bone biopsy \& placement of osteo-integrated implants. (A) Biopsy of the neomandible for histologic evaluation at 9 months post-reconstruction in conjunction to the sites of dental implants.

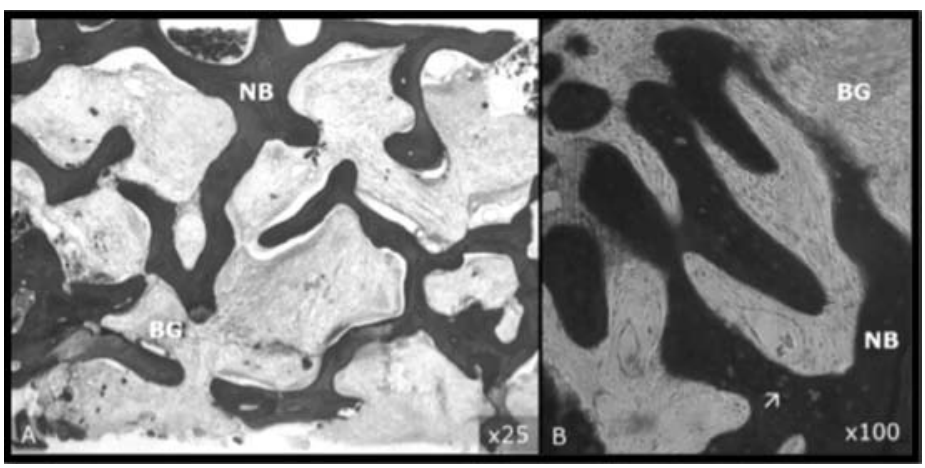

Figure 9. Histologic evaluation. (A\&B) Bone graft/matrix (NB-new bone, BG-bone graft) stained with trichrome showed presence of new mineralized tissues consisting of woven bone characterized by high number of distributed osteocytes and irregularly arranged fiber bundles within the new bone matrix osteocytes (arrow) evident of bone regeneration. (by Dr. Daniel Rothamel, University of Bonn, Germany, October 2011)

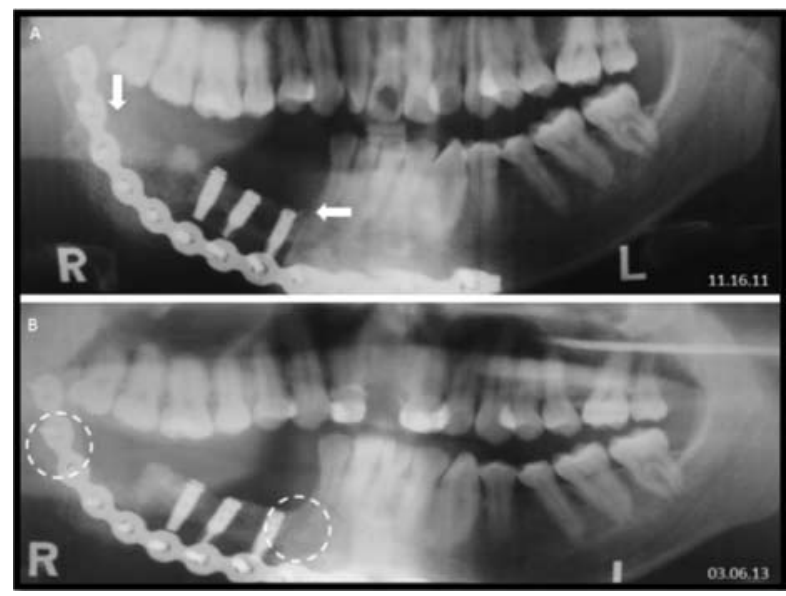

Figure 10. Latest radiographic series. (A) Gaps between the graft and the remaining mandible (arrows) 10 months after the procedure. (B) After 36 months, absence of previously noted gaps confirms continuous bone regeneration (circles).

\section{DISCUSSION}

Advancements in mandibular reconstruction have continued to develop over the past decades. The use of alloplastic materials like titanium plating implants for repairing mandibular defects provided patients with rapid rigid mandibular restoration but was limited by numerous complications such as infection, plate extrusion, and subsequent failure. Recently, the concept of distraction osteogenesis, involving bone distraction with an external mechanical device and progressive lengthening of the bone to allow a gap of new bone during the consolidation phase has also been used for mandibular reconstruction but has been limited by poor scar formation, delayed return to function, and inadequate formation of desired bone length. ${ }^{8}$ The advent of microvascular reconstructive surgery enabled the transfer of vascularized osseous flaps with the most commonly used fibular free flap showing superior results over non-vascularized bone transfer and better quality of life outcome. However, it did not remain free of donor-site morbidities. ${ }^{9}$

Tissue engineering led to the development and use of bone grafts that hold promise for the future of head and neck repair. ${ }^{10}$ Numerous clinical studies demonstrated the utility of tissue engineering in developing bone grafts for mandibular defect reconstruction. ${ }^{11}$ Such have already been widely used over the past decades in oro-maxillary and dental reconstruction, including the recombinant bone morphogenetic protein (rhBMP-2) that is now used with great success in cleft palate repair, alveolar ridge augmentation, and sinus lift procedures. ${ }^{12}$ Autogenous bone grafts derived from the patient work through osteogenesis, osteoinduction and osteoconduction. However, such are not recommended because apart from enabling a 
donor site morbidity-free technique, they are best harvested as microvascular flaps. Allogenic bone grafts on the other hand are cadaveric processed grafts that may be cortical, trabecular, or combined in composition and have both osteoconductive and osteinductive properties. Xenografts or processed animal bone graft are a subgroup of the synthetically manufactured alloplasts known to form new bones from their osteoconductive activities.

The use of autologous PRF is already widely used in combination with bone grafts for dental surgeries but not for large mandibular defects. ${ }^{13}$ Our reconstructive technique using bone grafts for large segmental mandibular defects emphasizes the important role of PRF with its intrinsic factors and leukocyte contents that release high amounts of growth factors such as TGBß1, PDGF-AB, VEGF and matrix glycoproteins. ${ }^{14}$ Collagen membrane used to envelop the entire recipient site creates a membrane barrier to prevent the growth of soft tissues and allow angiogenesis within the neomandible. ${ }^{15}$ Fascia lata may be used as a membrane barrier but defeats the authors' goal of an absolute donor site morbidity-free procedure. Overall, this particular technique along with gentle tissue handling and avoidance of oral cavity contamination for reconstructing large mandibular defects has been found to enhance bone regeneration capable for osteo-integrated dental implantation.

Generally, the harvesting and processing of autologous PRF is simple and inexpensive. Its use with bone grafts is a good substitute for segmental mandibular reconstruction in patients with contraindications to free flap procedures or in cases where patients simply wish to be free from any donor-site morbidity. However, this technique is limited to defects secondary to trauma and ablation of benign conditions as bone regeneration is expected in approximately 6 to 9 months. Mandibular defects following resection of malignant oral neoplasms are still best reconstructed with fibular free flaps as radiation therapy is warranted at the soonest possible time. Histologic validation of bone regeneration and osteoblastic activity index for the 13 other cases performed by the authors using this particular technique necessitates bone research centers that are capable of advanced bone analysis, and none are locally-available at this time. Meanwhile, this technique of combining autologous PRF in bone grafting remains an innovative and invaluable option for mandibular reconstruction today.

\section{REFERENCES}

1. Spiegel JH, DeRosa J. Mandibular Reconstruction. In: Lalwani AK, ed. Current diagnosis \& treatment in otolaryngology-head \& neck surgery. 2nd ed. New York, New York: McGraw-Hill Medical; 2008:367-373.

2. Hidalgo DA, Rekow A. A review of 60 consecutive fibula free flap mandible reconstructions. Plast Reconstr Surg. 1995 Sep;96(3):585-96; discussion 597-602.

3. Lohan DG, Tomasian A, Krishnam M, Jonnala P, Blackwell KE, Finn JP. MR Angiography of Lower Extremities at $3 \mathrm{~T}$ : Presurgical Planning of Fibular Free Flap Transfer for Facial Reconstruction. AJR Am J Roentgenol. 2008 Mar; 190(3):770-6.

4. Del Corso M, Vervelle A, Simonpieri A, Jimbo R, Inchingolo F, Sammartino G, et al. Current knowledge and perspectives for the use of platelet-rich plasma (PRP) and platelet-rich fibrin (PRF) in oral and maxillofacial surgery part 1: Periodontal and dentoalveolar surgery. Curr Pharm Biotechnol. 2012 Jun;13(7):1207-30.

5. Dohan DM, Choukroun J, Diss A, Dohan SL, Dohan AJ, Mouhyi J, Gogly B. Platelet-rich fibrin (PRF): a second-generation platelet concentrate. Part III: leucocyte activation: a new feature for platelet concentrates? Oral Surg Oral Med Oral Pathol Oral Radiol Endod. 2006 Mar; 101(3):e51-5.

6. Choukroun Jl, Braccini F, Diss A, Giordano G, Doglioli P, Dohan DM. nfluence of platelet rich fibrin (PRF) on proliferation of human preadipocytes and tympanic keratinocytes: A new opportunity in facial lipostructure (Coleman's technique) and tympanoplasty?. Rev Laryngol Otol Rhinol (Bord). 2007;128(1-2):27-32.

7. Schwarz F, Rothamel D, Herten M, Sager M, Becker J. Angiogenesis pattern of native and crosslinked collagen membranes: an immunohistochemical study in the rat. Clin Oral Implants Res. 2006 Aug;17(4):403-9.

8. Mehta RP, Deschler DG. Mandibular reconstruction in 2004: an analysis of different techniques. Curr Opin Otolaryngol Head Neck Surg. 2004 Aug:12(4):288-93.

9. Bozec A, Poissonnet G, Chamorey E, Casanova C, Vallicioni J, Demard F, et al. Free-flap head and neck reconstruction and quality of life: a 2-year prospective study. Laryngoscope. 2008 May; 118(5):874-80.

10. Torroni, A. Engineered bone grafts and bone flaps for maxillofacial defects: state of the art. Oral Maxillofac Surg. 2009 May;67(5):1121-7.

11. von Wilmowsky C, Schwarz S, Kerl JM, Srour S, Lell M, Felszeghy E, et al. Reconstruction of a mandibular defect with autogenous, autoclaved bone grafts and tissue engineering: An in vivo pilot study. J Biomed Mater Res A. 2010 Jun 15;93(4):1510-8.

12. Herford AS, Boyne PJ. Reconstruction of mandibular continuity defects with bone morphogenetic protein-2 (rhBMP-2). J Oral Maxillofac Surg. 2008 Apr;66(4):616-24.

13. Dohan DM, Choukroun J. PRP, CPRP, PRF, PRG, PRGF, FC. How to find your way in the jungle of platelet concentrates? Oral Surg Oral Med Oral Pathol Oral Radiol Endod. 2007; 103(3): 305306.

14. Dohan Ehrenfest DM, de Peppo GM, Doglioli P, Sammartino G. Slow release growth factors and thrombospondin-1 in Choukroun's platelet-rich fibrin (PRF): a gold standard to achieve for all surgical platelet concentrates technologies. Growth Factors. 2009 Feb;27(1):63-9.

15. Hämmerle CH, Lang NP. Single stage surgery combining transmucosal implant placement with guided bone regeneration and bioresorbable materials. Clin Oral Implants Res. 2001 Feb;12(1):9-18. 